\title{
First ten years of active metamaterial structures with "negative" elements
}

\author{
Silvio Hrabar ${ }^{*}$ \\ University of Zagreb, Faculty of Electrical Engineering and Computing, Unska 3, Zagreb, HR-10000, Croatia
}

Received: 18 September 2017 / Accepted: 27 June 2018

\begin{abstract}
Almost ten years have passed since the first experimental attempts of enhancing functionality of radiofrequency metamaterials by embedding active circuits that mimic behavior of hypothetical negative capacitors, negative inductors and negative resistors. While negative capacitors and negative inductors can compensate for dispersive behavior of ordinary passive metamaterials and provide wide operational bandwidth, negative resistors can compensate for inherent losses. Here, the evolution of aforementioned research field, together with the most important theoretical and experimental results, is reviewed. In addition, some very recent efforts that go beyond idealistic impedance negation and make use of inherent non-ideality, instability, and non-linearity of realistic devices are highlighted. Finally, a very fundamental, but still unsolved issue of common theoretical framework that connects causality, stability, and non-linearity of networks with negative elements is stressed.
\end{abstract}

Keywords: Active metamaterial / Non-Foster / Causality / Stability / Non-linearity

\section{Introduction}

All materials (except vacuum) are dispersive [1,2] due to inevitable resonant behavior of electric/magnetic polarization. The simplest physical picture deals with mechanical inertia of polarized particles, caused by their finite mass. Due to inertia, the particles cannot respond instantaneously to an external electromagnetic field. Thus, there will be a phase lag/lead between particle movement and applied oscillating field. Clearly, this effect causes a change of relative permittivity and permeability with frequency (i.e. the dispersion). An electrical engineering model of this phenomenon comprises an RLC resonant circuit (Lorentz dispersion model with "parallel" $\left(f_{\mathrm{p}}\right)$ and "series" $\left(f_{\mathrm{s}}\right)$ resonant frequency [1,2], Fig. 1).

Here, the resonant energy re-distribution between electric and magnetic fields describes the mechanical inertia. Inspection of the Lorentz curve (Fig. 1) shows that the dispersion is negligible if a material is used far below its parallel resonant frequency $\left(f_{\mathrm{p}}\right)$. Actually, this is the case of most of ordinary dielectrics in radiofrequency and microwave regime.

However, if one wants to make use of phase lead effect (i.e. of the occurrence of negative or less-than-unity effective constitutive parameters: EpsilonNeGative, MuNeGative, EpsilonNearZero, and MuNearZero), the

\footnotetext{
* e-mail: silvio.hrabar@fer.hr
}

dispersion becomes significant. The same behavior applies for metamaterials, in which above effects occur to the resonant energy redistribution in some kind of an electromagnetic structure [2]. Thus, every passive material (or metamaterial) with negative or less-than-unity effective parameters is inevitably narrowband. This fact is mathematically expressed in the form of well-known energy-dispersion constraints $[1,2]$ :

$$
\frac{\partial[\varepsilon(\omega)]}{\partial \omega}>0, \frac{\partial[\mu(\omega)]}{\partial \omega}>0 .
$$

Very similar equations apply for any reactive element in circuit-theory (Foster's reactance theorem) [3,4]:

$$
\frac{\partial[X(\omega)]}{\partial \omega}>0, \frac{\partial[B(\omega)]}{\partial \omega}>0 .
$$

Here $\varepsilon$ and $\mu$ stand for permittivity and permeability while $X$ and $B$ are reactance and susceptance, respectively, symbol $\omega$ represents angular frequency.

Obviously, ordinary reactive circuit elements (capacitors and inductors) obey Foster's theorem. However, one might (hypothetically) define new "negative" (or "nonFoster") elements: negative capacitor $\left(C_{\mathrm{n}}<0\right)$ and negative inductor $\left(L_{\mathrm{n}}<0\right)$ (Fig. 2$)$.

Due to negative capacitance/inductance, the terminal current of these elements is negative (it flows outward from the positive terminal). Thus, a negative capacitor and a 


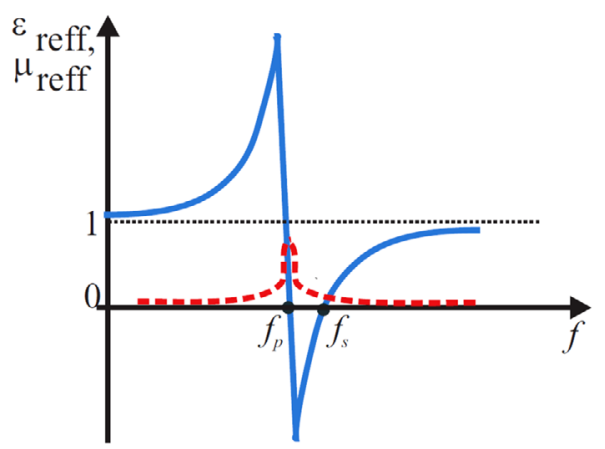

Fig. 1. Lorentz dispersion model. Solid: real part of relative permittivity/permeability, dashed: imaginary part of relative permittivity/permeability.

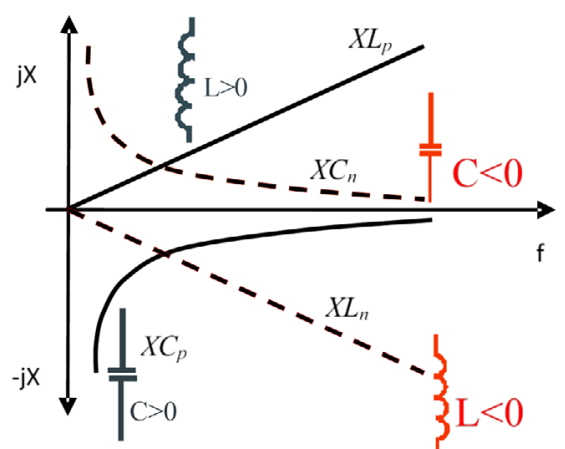

Fig. 2. Solid: reactance of positive (Foster) reactive elements, dashed: reactance of negative (non-Foster) reactive elements [20].

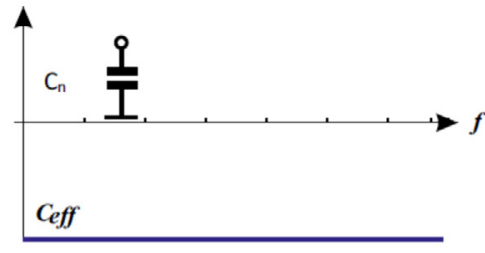

a)

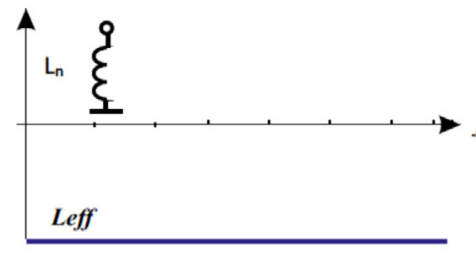

b)

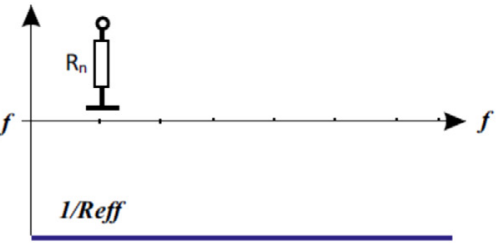

c)

Fig. 3. Linear dispersion-free models of ideal negative elements. (a) Negative capacitor, (b) negative inductor, and (c) negative resistor.

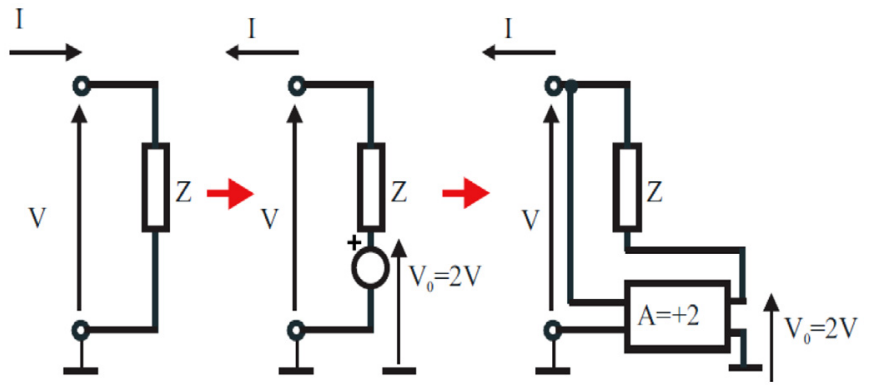

Fig. 4. Simplified explanation of the generation of negative impedance by a voltage-converting NIC [2].

negative inductor behave as (reactive) sources, i.e. they are inevitably active devices [2].

It is important to notice that energy-dispersion constraints (and Foster's theorem) have been derived assuming that there are no losses present $[1,3-5]$. So, strictly speaking, hypothetical negative resistor (an active element that delivers real power [4]) should not be termed as a "non-Foster element". However, one might say that a negative capacitor, a negative inductor, together with a negative resistor form a complete group of (hypothetical) "negative circuit elements". These ideal elements are postulated to be linear and dispersionless (Fig. 3).

In practice, "negative" elements are mimicked by appropriate electronic circuitry, so called "Negative Impedance Converters", [6,7] and "Negative Impedance Inverters", $[8,9]$, proposed more than 60 years ago. In essence, these are specially designed amplifiers with positive feedback. A positive feedback is needed in other to assure impedance sign flipping in the case of NICs $\left(Z_{\text {in }} \sim-Z_{\text {load }}\right)$ or impedance sign flipping accompanied with the impedance inversion in the case of NIIs $\left(Z_{\text {in }} \sim-1 / Z_{\text {load }}\right), Z_{\text {in }}$ and $Z_{\text {load }}$ being the input and load impedance, respectively. In order to get acquainted with the aforementioned principle, it is instructive to briefly explain the simplest voltage-conversion NIC (Fig. 4). The (grounded) load impedance that should be inverted $(Z)$ is shown in the left part of Figure 4. The load can be a lumped element of any type (a capacitor, an inductor, or a resistor). In order to invert the load impedance, one swaps the sign of the input current $(I)$. This is done by inserting an additional "dependent" voltage source of amplitude $2 \mathrm{~V}$ in series with impedance $Z$ (the middle part of Fig. 4). This dependent source is actually a voltage amplifier with gain equal to +2 (the right part of Fig. 4). The voltage drop across the impedance is now equal to $-V$ (the left electrode has potential of $V$ and the right one has potential of $2 V$ ). This causes the inversion of the impedance $Z_{\text {in }}$ (as seen from the input terminals):

$$
Z_{\text {in }}=\frac{V}{I}=\frac{V}{\frac{V-2 V}{Z}}=\frac{V}{-\frac{V}{Z}}=-Z .
$$

A more general equation that allows use of complex gain function of realistic active element can be found in $[2,6,7,10]$.

There are also some electronic elements (such as tunnel diodes and Gunn diodes [4]) that show negative resistance effects (but not negative capacitance or negative inductance!) per se. They again contain some kind of positive feedback, which occurs at a microscopic level due to 

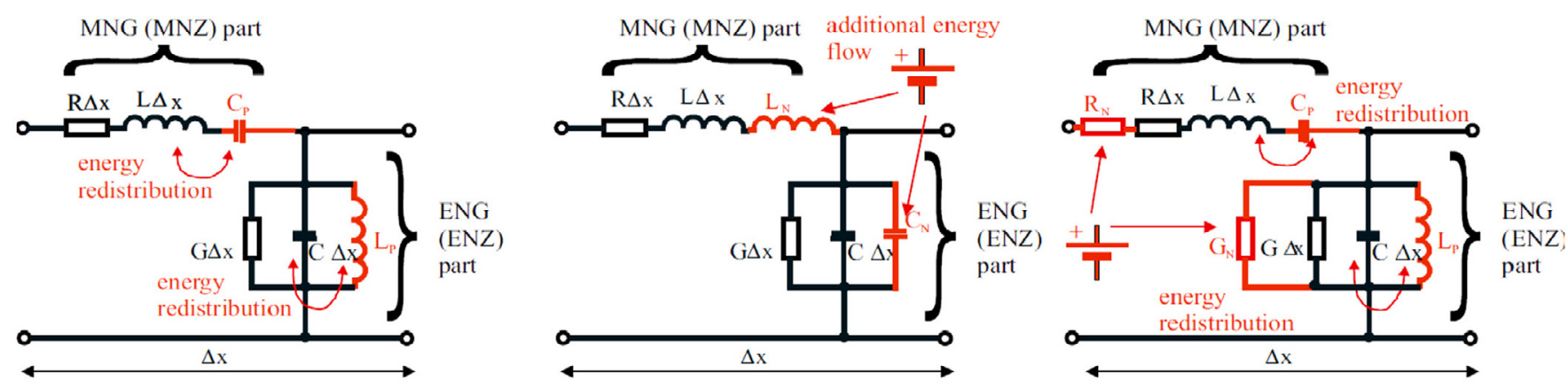

a)
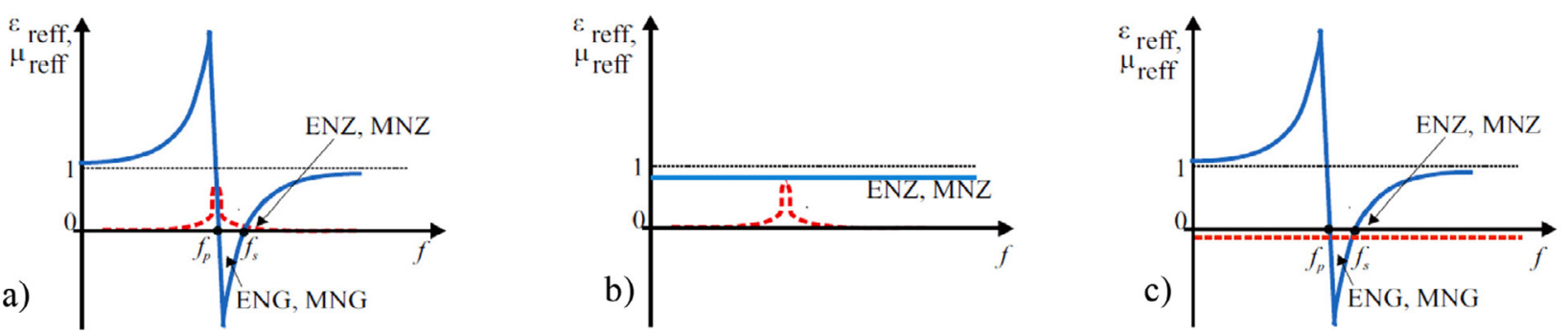

Fig. 5. Transmission-line models of different metamaterials (a) passive metamaterial, (b) active non-Foster-based metamaterial, and (c) active gain-based metamaterial.

background semiconductor physics. Traditionally, these elements have been used for construction of amplifiers and oscillators in the microwave community [4].

The first potential application of negative capacitors and inductors (that has been investigating for many years and it is still active nowadays) is broadband matching of small antennas [10]. Briefly, this matching relies on the compensation of frequency dispersion of an ordinary reactive network with the "inverse" dispersion of a "negative" non-Foster network. This compensation yields (theoretically) infinite bandwidth. Apart from matching, several (mostly theoretical) studies, that investigated the use of active elements in periodic structures for various microwave applications such as manipulation of bianisotropy [11] and emulation of perfectly matched layers (ideal absorbers) $[12,13]$, were published in the past.

Particularly interesting is an idea of "artificial atoms" that comprise circuits based on OPerational AMPlifiers (even including NIC circuits) [14]. Those "atoms" were proposed for construction of artificial structures that perform linear and non-linear shaping of a waveform of electromagnetic excitation signal.

The first paper (to the best of Author's knowledge) that theoretically discussed a possible use of active non-Foster elements for "dispersion compensation" in metamaterials was published in 2001 [15]. It analyzed the equations for polarizability of metamaterials based on an ensemble of short loaded dipoles and small loaded loops. In [15], it was shown analytically that a loading of a short dipole with a negative capacitance (instead of ordinary, positive inductance) should lead to the wideband dispersionless ENG behavior. Similarly, the loading of a small loop with a negative inductance (instead of ordinary, positive capacitance) should lead to the wideband dispersionless MNG behavior. In this case, the bandwidth would be limited only by the bandwidth of the realized non-Foster element itself. Thus, the inherent energy-dispersion constraints (1) would be bypassed.

Theoretical analysis from [15] initiated a new way of thinking and encouraged researchers all around the world to try to bring this interesting idea to reality. The field of active "negative" metamaterials has been growing rapidly. Nowadays it goes beyond broadband (almost dispersionless) operation, and includes power-flow reversal, nonlinear, and oscillatory behavior. This paper points out some of the most important (in Author's personal opinion) theoretical and experimental results obtained so far. On the other hand, it also tries to stress the "bottlenecks" of this technology, related to some very fundamental but still unclear issues.

\section{Evolvement of the research field and current state of the art}

\subsection{Active metamaterials and metamaterial-inspired devices}

The idea from [15] was extended using a transmission-line model of an isotropic material in [16]. Briefly, a standard transmission-line model comprises a series $R L$ branch (impedance of which is proportional to the complex permeability) and a shunt $G C$ branch (admittance of which is proportional to the complex permittivity) (the black elements in Fig. 5a). Here $L, C, R$, and $G$ stand for distributed inductance, capacitance, resistance, and conductance, respectively. In the case of ordinary passive MNZ or MNG metamaterials, one includes an additional lumped capacitor into the series branch (Fig. 5a). This causes a resonant redistribution of energy from the magnetic field (previously stored in a series distributed inductance) into 
the electric field (within additional lumped capacitor). Mathematically, new series combination possesses either zero impedance $\left(\mu_{\mathrm{r}} \sim 0\right.$, the MNZ effect at the resonant frequency) or a capacitive impedance $\left(\mu_{\mathrm{r}}<0\right.$, the MNG effect below the resonant frequency).

Similarly, one can include additional inductance into the shunt branch (the inductor in Fig. 5a) and achieve either the ENZ $\left(\varepsilon_{\mathrm{r}} \sim 0\right)$ or the ENG $\left(\varepsilon_{\mathrm{r}}<0\right)$ behavior. Of course, if both series and shunt braches are loaded (with series capacitor and shunt inductor, respectively), it is possible to achieve Double-Near-Zero $\left(\varepsilon_{\mathrm{r}} \sim 0, \mu_{\mathrm{r}} \sim 0\right)$ or DNG $\left(\varepsilon_{\mathrm{r}}<0, \mu_{\mathrm{r}}<0\right)$ behavior. At first sight, the circuit in Figure $5 \mathrm{~b}$ should lead to a single-resonance Drude dispersion model (the Lorentz model with $f_{\mathrm{p}}=0$ ). However, as detailed in [2], the inevitable "parasitic" capacitance of a realistic inductor and "parasitic" inductance of a realistic capacitor introduce additional resonance(s). Thus, the circuit (and the whole metamaterial) obeys the Lorentz dispersion (lower part of Fig. 5a). The main drawback of this (classical) passive metamaterial is a very pronounced inherent dispersion. From the circuit theory point of view, one may say that this dispersion occurs due to the basic difference in the frequency behavior of a capacitor and an inductor (solid curves in in Fig. 2).

However, if one (following a basic theoretical idea from [15]) replaces an additional shunt inductor with a negative capacitor (Fig. 5b), it will cause a (dispersionless) decrease of overall capacitance. Thus, resulting near-zero (or negative) behavior of equivalent permittivity will also be entirely dispersionless [17-20]:

$$
\varepsilon_{r}(\omega)=\left[C / \varepsilon_{0}-\left|C_{N}\right| /\left(\varepsilon_{0} \Delta\right)\right]
$$

Here $\Delta$ stands for the unit cell dimension. Clearly, very similar expression can be derived for the equivalent permeability in the case of added series negative inductor [17-20]:

$$
\mu_{r}(\omega)=\left[L / \mu_{0}-\left|L_{N}\right| /\left(\mu_{0} \Delta\right)\right]
$$

Thus, if negative elements are ideal, the real parts of effective permittivity, permeability will be "flat" with frequency (lower part of Fig. 5b). What is the background physics of this dispersionless behavior? Actually, the negative capacitor and negative inductor behave as frequency-dependent controlled sources [21,22] that supply additional currents to the positive elements. For instance, there is an additional current in the shunt branch (caused by the negative capacitor). This current causes faster charging of the distributed capacitance and, therefore, it decreases the overall effective capacitance (and effective permittivity). Instead of an energy redistribution from the electric field into magnetic field (or vice versa) used in passive metamaterial, here one introduces an additional energy flow from the active device (that has its own DC power supply).

The first experimental verification of above concept was the realization of a single ENZ non-Foster-based unit cell [20], previously proposed theoretically in [16]. The cell was constructed using NII FET-based negative capacitor incorporated into a 2D microstrip unit cell. Measurement of effective permittivity showed fairly constant ENZ behavior $\left(0.25<\varepsilon_{\mathrm{r}}<0.35\right)$ within the octave bandwidth $(1-2 \mathrm{GHz})[2,20]$. Measured results were also used as input data for $\mathrm{ADS}^{\mathrm{TM}}$ simulation of a proposed active $2 \mathrm{D}$ plasmonic cloak [20] and showed operating relative bandwidth of $100 \%$ (comparing to relative bandwidth of $20 \%$ in the case of a passive cloak).

Application of non-Foster elements in broadband cloaking was refined later in several studies. The analysis of a thin cylindrical cloak with rectangular patches, edges of which are loaded with negative capacitors showed significant bandwidth enhancement [23,24]. Along the same line, application of non-Foster elements (both negative capacitors and negative inductors) in an anisotropic cylindrical cloak was studied in [25], and a very wide cloaking bandwidth (1:5) was predicted. It is important to notice that all these studies on non-Foster cloaking were entirely analytical and numerical while experimental realization has not been reported so far.

The first practical 1D active ENZ metamaterial (an air transmission line loaded with three OPamp-based negative capacitors, as shown in Fig. 6a and b) was analyzed, built and tested in the lower $\mathrm{RF}$ regime $[2,26]$. The real part of effective permittivity (extracted from the measurements of a full scattering matrix) was found to be rather constant (it varied from 0.27 to 0.37 ), in the frequency range of $2-40 \mathrm{MHz}$ (bandwidth of more than four octaves), Figure 6c. This bandwidth is significantly wider than a bandwidth of any passive ENZ metamaterial. In addition, the propagation characterization in both frequency and time domain revealed that such a "non-Foster transmission line" supports counter-intuitive (but causal) superluminal phase and group velocities [26,27], Figure 6d. Later, similar experiments, based on an air transmission line loaded with three negative capacitors (constructed as Linvill's bipolartransistor-based floating NICS [7]), operating from 60 to $120 \mathrm{MHz}$ and from 50 to $220 \mathrm{MHz}$, were reported in [28] and [29], respectively. It is interesting that the authors in [29] verified "superluminal" propagation not only by extraction from measured scattering matrix, but also from near field measurements of the electric field in air above the transmission line. The longest (38 unit cells) "superluminal" non-Foster transmission line, operating from 42 to $60 \mathrm{MHz}$ has also been constructed and tested recently [30]. In this thesis, the author also conducted thorough analysis of the practical limitations on "superluminal propagation" in nonFoster transmission lines.

The examples reviewed so far used one-dimensional non-Foster metamaterial structures (i.e. transmission lines). The first attempt to directly apply the basic idea of an array of small antennas loaded with non-Foster elements in order to achieve $2 \mathrm{D}$ or $3 \mathrm{D}$ metamaterial was reported in [31]. Briefly, a $2 \times 2$ array of vertical dipoles loaded with negative capacitors, operating in the low RF frequency range (up to $50 \mathrm{MHz}$ ), was located within a simple parallel-plate capacitor that imposed periodic boundary conditions in the vertical direction. In such a way, a structure behaved as a "pile" with infinite number of layers, i.e. as a $2 \frac{1}{2}$ D structure. Equivalent permittivity was extracted from the measurement of capacitance of a parallel-plate capacitor. Obtained bandwidth $(10-20 \mathrm{MHz}$, 

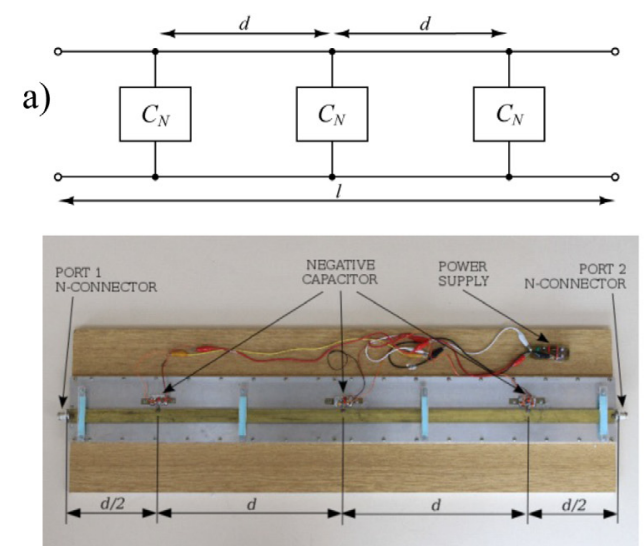

b)
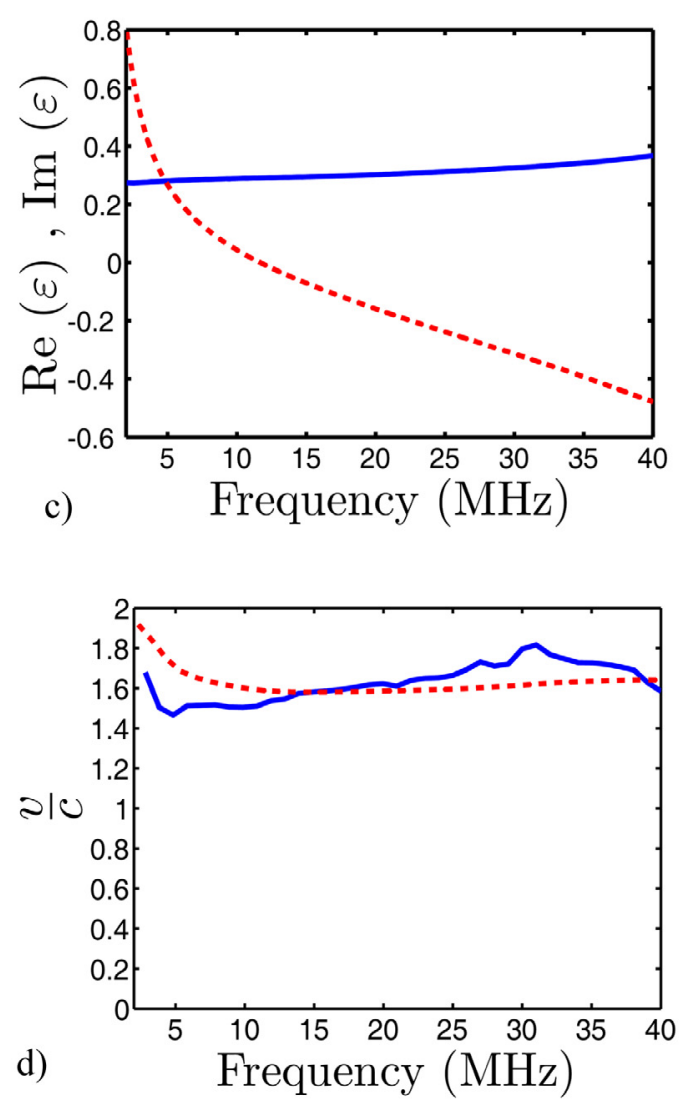

Fig. 6. The first non-Foster ENZ transmission line (1D nonFoster metamaterial) $([2,26,27])$. (a) Basic block-diagram, (b) a photo of manufactured prototype, (c) measured effective permittivity, solid-real part, dashed - imaginary part, and (d) measured signal velocities, solid - group velocity, dashed- phase velocity.

i.e. one octave) was again wider than the bandwidth of passive ENZ metamaterials. However, this bandwidth was clearly narrower than the bandwidth of a $1 \mathrm{D}$ active transmission-line-based ENZ metamaterial reported in [26]. This happened due to capacitive coupling between neighboring dipoles that cannot be compensated by nonFoster elements (a very similar effect, but within a different context, was discussed in [32]). Several similar designs of volumetric 3D metamaterials with negative elements were proposed theoretically in [33]. No experimental realization of a volumetric 3D non-Foster-based metamaterial structure has been published so far.

In recent years, the whole electromagnetic community has focused their research efforts on thin artificial sheets (so-called metasurfaces [34]), which are 2D equivalents of metamaterials. An obvious advantage of this approach is a smaller number of unit cells (that results with lower losses) and very simple fabrication. There are some reports on nonFoster-based metasurfaces, as well. The broadband nonFoster-based artificial magnetic conductor (high-impedance metasurface) was reported in $[35,36]$. It was based on an array of small (in terms of wavelength) patches with a central grounded pin ("mushrooms"), mutually connected with negative inductors fabricated in microelectronic technology. The measurements, performed in a specially designed tapered coaxial TEM cell verified behavior very similar to that of an ideal magnetic conductor within 200-400 MHz bandwidth. In addition, the broadband nonFoster-based metasurface with very low dispersion was reported in [37]. Two different versions of metasurfaces, containing $7 \times 10$ unit cells with patches were manufactured. Non-Foster $L C$ circuits were connected across the gaps of neighboring patches, compensating the dispersion of a bare array. The near-field measurements showed almost dispersionless refractive index of 1.3 and 1.5, over the fractional bandwidth of $56 \%(450-800 \mathrm{MHz})$. The last example of a non-Foster metasurface is a broadband absorber [38]. It comprised a thin (less than $\lambda / 100, \lambda$ being the signal wavelength) array of vertical wires periodically loaded with negative $L C$ circuits (implemented using OPamp technology). This array was used as a termination of a simple TEM transmission line, a reflection coefficient of which was measured by a vector network analyzer. Measured return loss was better than $10 \mathrm{~dB}$ across a $150-950 \mathrm{MHz}$ band, indicating broadband absorption. Additional measurements and discussion on matching properties of similar type of absorber (with magnetic material) were also published very recently [39].

For the sake of completeness, one should look at "metamaterial-inspired" applications that, instead of employing negative elements in metamaterials/metasurfaces extend basic principles to some other field. The first example is the enhancement of properties of small antennas by adding non-Foster elements into the antenna structure and, therefore, changing its current distribution (it is worth stressing that this approach is radically different from "external" active matching). There are several both theoretical [40] and experimental studies [41-44] of this idea. The second example is a squint-free leaky wave (LW) antenna based on a transmission line periodically loaded with negative capacitors, proposed in [45] and later analyzed in [46,47]. This type of non-Foster antenna has been analyzed theoretically and numerically, while there is no experimental investigation reported so far.

\subsection{Technological issues}

Let us point out some of the important issues in the design of non-Foster-based unit cell per se. Firstly, the cut-off frequency of an active element has significant impact on the 
achievable operation bandwidth $[2,20]$. The cut-off frequency should be significantly higher than the required highest operating frequency of the negative capacitor. As a rule of thumb, one could say that the cut-off frequency should be at least ten times higher than the maximal operating frequency [20]. This comes from a very simple fact that the operation of all NIC and NII circuits is based on either voltage or current inversion. The inversion requires a phase shift of zero degrees between the input and the output of the circuit. Nearly zero phase shift can be achieved only at very low frequencies, for which the transit time across the active device can be neglected. Although not obvious at first sight, the same requirement on cut-off frequency applies to a variant of NII circuits that (instead of a classical amplifier) employs a "converting network" with positive and negative resistors [8].

The second important issue is the trade-off between different technologies (BJT, FET, OPamp, customdesigned integrated circuit, and special microwave elements such as a resonant tunnel diode). Generally speaking, use of OPamps is limited by the maximal operating frequency, which for the fastest devices nowadays lies around $2 \mathrm{GHz}$. Thus, the maximal frequency of an associated OPamp-based negative element could be couple hundreds of $\mathrm{MHz}$. It is a rather low frequency that obviously excludes microwave applications. However, the OPamp-based design is very simple, without complicated DC bias networks. The problem of occurrence of parasitic capacitances and inductances is not pronounced and the repeatability of manufactured circuits (even in the case of simple "hand-crafting") is excellent. Therefore, OPamp technology is a very convenient choice for constructing proof-of-concept demonstrators.

The use of discrete BJT and FETs assures higher operating frequency of associated negative elements, which is currently around $1-2 \mathrm{GHz}[20,48]$. On the other hand, there are problems with "parasitics", the influence of an unwanted phase shift of connecting leads on the circuit parameters is rather pronounced, and rather complicated active biasing networks are often needed $[2,20]$. Thus, the use of discrete BJT and FET elements yields broad bandwidth, but it is accompanied with rather complicated design and challenging repeatability (especially in the case of "hand-crafting").

The next possibility is the use of negative elements based on specially designed integrated circuits. This solution offers a very high operating frequency that lies in the UHF [49] or even in the microwave regime [50-52] and parasitic effects are negligible. The downside is that the microelectronic technology is (initially) very costly and not available for many research groups. There is also a rather interesting approach based on one-port negativeresistance microwave elements such as resonant tunnel diodes as a part of abovementioned resistive converting network [53]. This approach offers high operating frequency (in the $\mathrm{GHz}$ regime) and a design of mild complexity.

Yet another very interesting approach employs similarity between NeGativeDelay circuits (the circuits that employ phenomenon of anomalous dispersion in the vicinity of the loss line) and non-Foster behavior [54-56]. It employs a resonant structure with pronounced losses, which are needed for the NGD effects. The losses are compensated by the inclusion of an integrated amplifier. In such a way, it was shown possible not only to build unidirectional non-Foster-like unit cells, but also to construct a practical squint-free steerable antenna array operating within $1-1.5 \mathrm{GHz}$ band [56].

In addition, there are several very recent examples of digital non-Foster elements [57,58]. They are based on monitoring original input voltage (or current) signal via $\mathrm{A} / \mathrm{D}$ converter, analyzing it by processing circuitry, and modifying it by the injection of an additional signal via $\mathrm{D} / \mathrm{A}$ converter. By doing this, any value of positive/ negative impedance can be emulated. This approach is very versatile and almost completely immune to component ageing and imperfections. The drawback is a low operating frequency (around $100 \mathrm{MHz}$ ), constrained with state of the art digital signal processing hardware.

In all above examples, it is in principle, possible to add tunability and re-configurability features. In [33], several simple OPamp-based demonstrators of negative capacitors and inductors were constructed using standard electronic tuning elements (the varactors and PIN diodes). In this way, it was possible to build the ENZ unit cell with tunable relative permittivity from 0.2 to 0.8 , and the MNZ unit cell with tunable relative permeability from 0.2 to 0.5 , both operating across very wide bandwidth $(100 \mathrm{kHz}-700 \mathrm{MHz})$. Similar approach that used an FET-based variable resistance was applied in a microelectronic realization of negative inductance [49]. Achieved tuning range was -64 to $-40 \mathrm{nH}$, within $400-600 \mathrm{MHz}$ band.

Apart from the use of negative capacitors and negative inductors, several research groups proposed the use of negative resistors ("active gain-based metamaterial structures") $[5,18]$. Here, the main idea is not dispersion management but rather the compensation of inherent losses. Simply, a negative resistor is incorporated either in series or shunt branch of a given transition line (i.e. metamaterial structure) (Fig. 5c) [5]. Of course, the negative-resistance elements also have their own DC sources, so there is again an additional energy flow into the metamaterial structure. The advantage of this approach is a relatively simple design (particularly if one uses one-port devices as tunnel diodes). In addition, negative-resistance devices are available across almost whole $\mathrm{RF}$ and microwave regimes.

Finally, several studied used embedded amplifiers for gain compensation and dispersion management of inclusion-based metamaterials $[59,60]$. The embedded amplifier might be considered as a special case of (unidirectional) negative resistor (similarly to already discussed NGD unidirectional negative-capacitor unit cell [54-56]). The approach in [59] employs a "sensing and intervening" structure. The "sensing" (receiving) loop is connected to the amplifier that modifies the signal (both the amplitude and phase) and radiates it back into the metamaterial structure via "intervening" (transmitting) loop. This method yielded a low loss MNG metamaterial operating in UHF (500$800 \mathrm{MHz}$ ) regime with a bandwidth of $25 \%$. Improved version reported in [60] (with additional split-rings and voltage-controlled phase shifter) showed zero-loss with tunable magnetic response. 


\subsection{Stability}

The most important problem that still prevents widespread application of metamaterial structures with negative elements is the stability management [2]. All negative elements (both non-Foster capacitors and inductors as well as negative resistors) employ some kind of a positive feedback, which in some case leads to instability. This issue is still not well understood, which makes a design of stable non-Foster circuits extremely challenging.

Many methods of stability prediction that are routinely used in microwave engineering fail in the case of non-Foster networks. A detailed review of this problem is given in [61-64]. Briefly, there are two important issues: selection of the domain of analysis and the representation of an analyzed network. The simplest approaches use the analysis in phasor domain (steady-state analysis with real frequencies). This approach neglects the transient response that is crucial in non-Foster circuits, so it should not be used. Thus, stability analysis in the Laplace domain (with complex frequency $s=\sigma+j \omega$ ) or in the time domain is a first prerequisite (necessary, but not always sufficient!) for the reliable stability prediction. The second prerequisite is associated with the representation of an analyzed network. The methods that reduce the network to the equivalent "black box" and then look at the properties of the "black box" input port impedance (or reflection coefficient) may fail. Several examples of such failure are given in [2,62-64], for the case of the widely used Rollet method. For instance, the modulus of the reflection coefficient of an ideal negative capacitance is one, as is the modulus of an ordinary positive capacitance. Looking at this result, one could (wrongly) conclude that a pure negative capacitor is a stable device. However, a very simple and intuitive transient analysis [65] clearly shows an unbounded growth of voltage across the negative capacitance, i.e. instability. This happens due to the inherent poles located in the Right-Hand-Side of a complex plane, the existence of which cannot be inferred just from the analysis of the "black box" input port reflection coefficient (the existence of so-called "hidden modes") [62-64]). In order to avoid problems with hidden modes one should use the method (either in the Laplace domain or in the time domain) that takes into account all the meshes and nodes of the analyzed network. Thus, only the methods that use Laplace domain (or time domain) and take into account all the meshes and nodes of the analyzed network (analysis of system determinant [62,63], Normalized Determinant Function [66], Nyquist method [63], etc.) are always reliable and cannot result with incorrect stability prediction.

Another important fact is that stability does not depend only on the non-Foster element but also on the other elements in a given network [61]. Usually, it is believed that it is enough to fulfill widely accepted stability criterion that the overall "mesh" capacitance or inductance must be positive number. This criterion was discussed in details in $[67,68,72]$. A common way of achieving this "compensation" is the insertion of an additional positive element (the so-called "swamping element") in series or in parallel to negative element. Therefore, a new equivalent element (a combination of

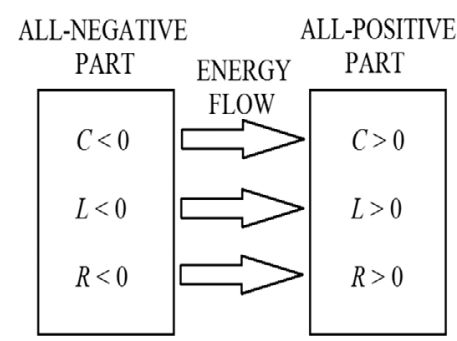

Fig. 7. Stable structure based on a mixture of "positive" (passive) and "negative" (active) parts.

positive and negative element) behaves as an ordinary positive element. Since all positive elements are stable, it appears logical that a new combination should always be stable. Actually, that is the reason why early efforts dealt only with near-zero (ENZ/MNZ) metamaterials (negative (ENG/MNG) metamaterials violate above stability criterion) [19,20,26,27].

Some time ago, it was found that it might be possible to build a stable ENG, MNG, or DNG non-Foster metamaterial [33,69,70] (although that appeared impossible in previous studies $[19,20,26,27])$. As shown in [33,61,69], a network comprising solely negative elements is inherently stable (as is an ordinary network that contains solely positive elements). In such a structure, the power flow is reversed (the energy travels towards the source). First experimental confirmation [33] demonstrated a stable negative RLC circuit with reversed power flow. Following these initial efforts, one may include negative resistors into a non-Foster circuit obtaining an "all negative part" connected to an "all positive part" (ordinary network with positive R,L,C elements) (Fig. 7). This system is stable providing that overall resistance, capacitance and inductance is negative. The experiment in [33] revealed that energy indeed flows from the negative part into the positive part of the system. It is important to notice that this system contains only lumped elements without any transmission line.

However, there are (at least) two issues that put some doubts on the above simple stability analysis based on overall positive (or overall negative) capacitance and inductance. Firstly, it was shown that the presence of "swamping" elements is not always necessary [62]. For instance, a simple equivalent circuit of an ideal transformer (that is, of course, an unconditionally stable device) contains some negative elements without "swamping" neighbors [62]. Similar example that involves an equivalent circuit of lattice unit cell of periodic structure was shown in [55]. In addition, one should not forget that the overall "mesh" criterion was derived postulating that non-Foster elements are entirely dispersionless (Fig. 3). Unfortunately, ideal dispersionless negative elements violate basic physics (causality and linearity $[21,22,70])$, and, therefore, they cannot exist in practice. Indeed, the gain of every realistic active element used in "negative" circuitry (regardless of used technology) is always band-limited. Hence, realistic negative elements will always be band-limited, dispersive devices (Fig. 8). 


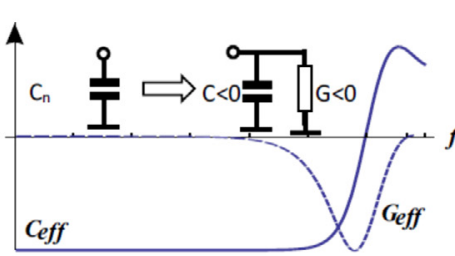

a)

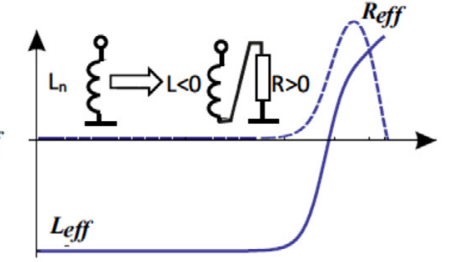

b)

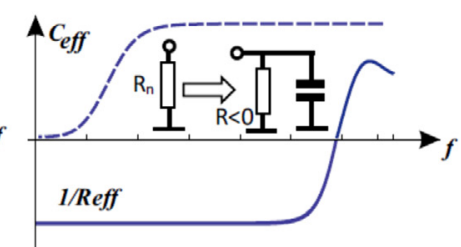

c)

Fig. 8. Linear (dispersive) models of realistic negative elements. (a) Negative capacitor, (b) negative inductor, and (c) negative resistor.

Furthermore, required negative reactance is always accompanied by occurrence of unwanted resistance (either positive or negative), Figure 8. This fact may be interpreted as a special case of Kramers-Kronig relations (or, in a broader sense, of causality [1]). So, in practice, it is impossible to generate a pure negative capacitance or a pure negative inductance. This unfortunate fact makes stability prediction extremely challenging.

It is interesting that some theoretical studies predicted serious stability problems in early 1D non-Foster metamaterials [71]. On the contrary, there are several reports on successful practical realizations [19,20,26,27]. This again emphasizes that the stability issue in non-Foster networks is still not well-understood.

Very recent study [72] of negative capacitors shed some new light on stability. It was shown that the stability criterion of a positive "mesh" capacitance fails for the combination of positive and ideal negative capacitor, connected via a segment of transmission line. Such a network is always unstable, for any line length (different than zero) and capacitance values of positive and negative capacitor. These problems are caused by an unstable pole that is located on the real axes in the RHS of a complex plane. It is rather counter-intuitive since the real pole supports zero-frequency ("DC") exponentially growing signals. This appears as "bad news" since all kinds of negative elements (including microelectronic realizations) will inevitably have some connecting leads of finite length. It could mean that these systems would always be unstable. Surprisingly, the limited bandwidth of a realistic negative capacitor (i.e. that inherent dispersion, Fig. 8) may, for some design parameters, suppress the occurrence of the real pole. In other words, non-ideality of the negative element, in some case, assures stable operation. This might be the explanation why some experimental studies reported stable operation [19,20,26,27] although the associated theoretical analysis (based on ideal elements) predicted instability. Even more surprisingly, in some cases, a non-ideality of used elements can assure stable operation of a network with negative overall capacitance or inductance [72]. This novel effect can be used for the construction of stable dispersionless ENG/ MNG/DNG metamaterials.

Concerning the stability of $2 \mathrm{D}$ structures with negative elements, there are very few papers published so far. In [73-75], the authors analyzed a 2D array of small loops loaded with negative elements (2D magnetoinductive metamaterial) and investigated the influence of various design parameters on stability. This analysis was done using an equivalent circuit of generalized magnetoinductive metamaterial and applying Nyquist criterion.

Along a similar line of thought, the authors in [76] analyzed incorporation of inclusions loaded with negative elements both into generalized multi-layer 2D structures and into a simple patch array. Both the analytical investigation based on equivalent circuit and full-wave numerical results were presented.

The stability of active gain-based metamaterial structures appears to be a slightly simpler task [18]. Here, stability primarily depends on the remaining negative resistance within unit cell ("left" after loss compensation), and the overall number of gain cells in the structure. These issues can be analyzed using standard, well-developed (and well-understood) theory of reflection amplifiers and negative-resistance oscillators [4].

\section{Open questions, new ideas, and future trends}

\subsection{Tailoring stability properties by "exotic" design}

Obviously, it would be convenient to avoid occurrence of a real pole in a non-Foster network and, therefore, assure stable operation. As indicated in [72] it could be possible by a "bandpass" design of the negative element. This proposal is similar to stability analysis of a hypothetical non-Foster element that behaves as a negative capacitance within a finite frequency band, (outside of this band it behaves as ordinary inductance [77]). The "bandpass" approach might be useful for construction of stable broadband ENG, MNG and DNG metamaterials. Whether these "bandpass negative elements" (which, strictly speaking, are not negative capacitors or inductors, anymore) can indeed be constructed in practice, is open question. In the University of Zagreb Group, the design and testing of a low-frequency experimental demonstrator of a "bandpass negative capacitor" is under progress.

Another open question is whether "all negative metamaterials" with reversed power flow, based on negative capacitance, inductance and resistance [33,69] (Fig. 7) can be brought to real engineering applications. Although the basic idea was developed using ideal dispersionless elements (that cannot exist in practice), some recent studies indicate that negative power flow is possible with realistic elements, as well [70]. 


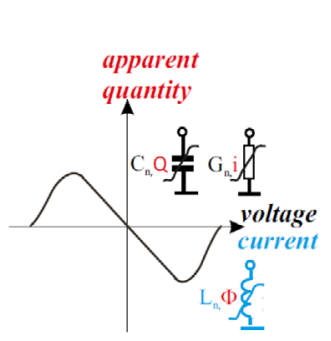

a)

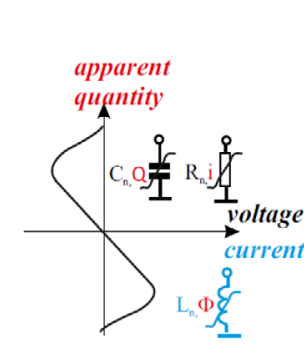

b)

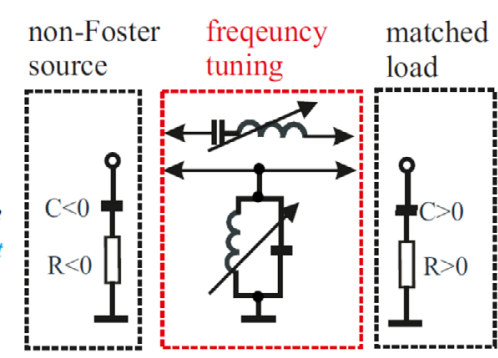

C)

Fig. 9. (a) Generalized N curve of a non-linear negative element [70], (b) generalized S curve of a non-linear negative element [70], and (c) a concept of self-oscillating tunable non-Foster antenna-transmitter [78,79].

\subsection{Non-linear and oscillatory behavior for active resonator/antenna/metasurface applications}

Yet another open question is whether one can always neglect the non-linear properties of negative elements. Actually, all previous examples presumed linear operation. However, exponential growth predicted in [72] will certainly drive the circuitry into a non-linear regime. Similar behavior is well understood in the case of negative-resistance oscillators (well-known "N" and "S" curves, [4]). Recently, it was shown possible to construct similar curves by introducing "apparent electric charge" and "apparent magnetic charge" ("apparent flux") for the cases of negative capacitor and negative inductor, respectively [70], Figure $9 \mathrm{a}$ and b. By the use of generalized " $\mathrm{N}$ " and "S" curves it is possible to predict change of generated negative capacitance and inductance as a function of input signal magnitude. It would certainly help in accurate design of self-oscillating, broadband non-Foster radiating structures, introduced experimentally in $[78,79]$. Actually, these are unstable negative structures, impedance of which is a "negative image" of the antenna impedance (Fig. 9c). In this way, it is possible to achieve a tunable and perfectly matched transmitter-antenna system, or even a metasurfacebased transmitting array across the 1:10 bandwidth [79]. This approach is fundamentally different from the ordinary active antenna since the dispersion of antenna impedance does not influence matching. Particularly important is an extension of this approach to resonatorfree self-oscillating metasurface tuned by injection locking from an external beam, within a very broad bandwidth [78,79]. Moreover, the non- linearity can also be used for up/down conversion into different frequency bands. In all these applications, inherent instability of negative capacitors and inductors is an advantage, rather than a drawback.

Furthermore, there is a similarity between metamaterials based on "negative elements" and PT-symmetry systems, as shown in [80]. This may lead to development of novel distributed, broadly tunable oscillators and antenna transmitter systems. Observed similarity can even be extended to recently proposed "EM field teleportation" systems [81], which again highlights importance of reversed power-flow from $[33,69]$.

\subsection{Adding bianisotropic (reciprocal or non-reciprocal) behavior}

The concept of bianisotropy, although being very old, has been applied to transmission-line-based metamaterials very recently. An interesting example is experimental omega transmission line [82], which is actually a broadband version of the well-known omega medium. The basic idea is an additional cross-coupling between series and shunt braches of the transmission line via transformer [17] (or, equivalently, the cross-coupling between orthogonal components of electric and magnetic field vectors of a plane wave). This principle was further extended in [83] using active coupling (via gyrator) enabling moving-media behavior. The same idea may be applied for both gain-based and non-Foster-based active metamaterials. It would provide stable bianisotropic (both reciprocal and non/reciprocal) behavior across a wide RF band. Particularly interesting would be the combination of negative elements with a metasurface with exotic boundary conditions such as the DB boundary $[84,85]$. This might be a way towards implementation of an active metasurface that emulates generally linear boundary conditions [86].

\subsection{Basic theoretical issues that are still unclear}

It should be stressed that the progress in all practical engineering applications of active metamaterials/metasurfaces based on negative elements is rather challenging due to basic, but still unsolved theoretical problem. Namely, a construction of a unified theoretical framework that connects causality, stability and non-linearity of "negative elements". Particularly, it was shown that there are many similarities between negative group delay, "superluminal" effects, and non-Foster behavior in steady-state conditions [70]. But, a theoretical link that takes stability into account (i.e. the connection in Laplace domain) is unclear.

In addition, one intuitively expects that stability and causality are somehow connected. However, at the moment it is not even clear whether Kramers-Kronig relations can always be applied for testing the causality of active metamaterials or not $[87,88]$. The problem is the well-known prerequisite for the use of Kramers-Kronig 
relations. It states that there should not be any poles in the RHP of a complex plane (assuming engineering $e^{j \omega t}$ time convention). Thus, for a causality prediction of some system, one should a priori presume its stability. If stability cannot be presumed, Kramers-Kronig relations should not be used. As a solution, one may turn to general relativistic causality [87]. However, again, it is not clear how to apply this test in the case of systems with negative elements. It is very important to stress that a missing theoretical framework is not just an "academic issue". On the contrary, it has very strong practical consequences. If one cannot apply some tool for causality testing, one cannot predict whether expected effects are indeed physically feasible or not [22]. If a unified theoretical framework were available, it would be possible to better understand effects that currently slow down the development of the field of active negative-elements-based metamaterials.

\section{Conclusion}

The field of active metamaterial structures based on "negative circuit elements" (negative capacitors, negative inductors and negative resistors) has grown extensively within its first decade. Specifically, non-Foster broadband metamaterial structures have been improved and their properties are becoming closer to those needed in realword applications. In addition, there are some novel ideas that go beyond idealistic impedance negation. These make use of inherent non-ideality, instability and nonlinearity of realistic devices for future self-oscillating and self-mixing antenna applications. So, the future of the field appears exciting. It is interesting that, in spite of a great progress and many practical demonstrations of novel effects, some very fundamental issues are still unclear. Thus, the development of a unified theoretical framework that connects causality, stability, and nonlinearity is probably one of the most important future tasks.

Part of this material reviews results achieved by the Author's group at the University of Zagreb, Faculty of Electrical Engineering and Computing. These results are based upon the work on the following projects: "Electromagnetic Metamaterials and Nano-electromagnetics", MZT, Contract 036-0361-566-1571; "Broadband Epsilon-Near-Zero (ENZ) and Mu-Near-Zero (MNZ) Active Metamaterial", EOARD/AFRL, Contract FA 8655-10-13030; "Passive and Active Metamaterial Structures for Guiding, Scattering and Radiation of Electromagnetic Energy", UKF, Contract 09/13; "Active Reconfigurable Metamaterial Unit Cell Based on Non-Foster Elements", EOARD/AFRL, Contract FA8655-12-1-2081; "Non-Foster Source-load Networks and Metasurfaces", EOARD/AFRL Contract FA9550-15-1- 0120; "Active Ultra-wideband Transmitter-antenna System", HAMAG-BICRO Contract POC6_1_233.

\section{References}

1. G.L.D. Landau, E.M. Lifshits, Electrodynamics of Continuous Media, 2nd edn. (Buttterwoth Heinmann, Oxford, 2002)
2. S. Hrabar, I. Krois, I. Bonic, A., Kiricenko, E.U. Munoz, Broadband Epsilon-Near-Zero (ENZ) and Mu-NearZero (MNZ) Active Metamaterial, Final Report for Contract FA 8655-10-1-3030, EOARD/AFRL, August 2011

3. R.M. Foster, A reactance theorem, Bell Labs Syst. Tech. J. 3, 259 (1924)

4. D.M. Pozar, Microwave Engineering (Willey, New York, 1998)

5. Q. Tang, Active Metamaterial: Gain and Stability, and Microfluidic Chip for $\mathrm{THz}$ Cell Spectroscopy, $\mathrm{PhD}$ thesis, The University of Arizona, 2017, http://hdl.handle.net/ $10150 / 623025$

6. J.L. Merrill, Theory of the negative impedance converter, Bell Sys. Tech. J. 30, 88 (1951)

7. J.G. Linvill, Transistor negative impedance converters, Proc. IR 41, 725 (1953)

8. L. Verman, Negative circuit constants, Radio Eng. Proc. Inst. 19676 (1931)

9. B. van der Pol, A new transformation in alternating-current theory with an application to the theory of audition, Radio Eng. Proc. Inst. 18, 220 (1930)

10. A.K. Perry, Broadband antenna systems realized from active circuit conjugate impedance matching, AD-769 800, Naval Postgraduate School, 1973, http://www.dtic.mil/dtic/tr/ fulltext/u2/769800.pdf

11. F. Auzanneau, R.W. Ziolkowski, Theoretical study of synthetic bianisotropic materials, J. Electromag. Waves 12, 353 (1998)

12. R.W. Ziolkowski, The design of Maxwellian absorbers for numerical boundary conditions and for practical applications using engineered artificial materials, IEEE Trans. Ant. Propagat. 45, 656 (1997).

13. S.A. Tretyakov, T.G. Kharina, The perfectly matched layer as a synthetic material with active inclusions, Electromagnetics 20, 155 (2000)

14. F. Auzanneau, R.W. Ziolkowski, Artificial composite materials consisting of nonlinearly loaded electrically small antennas: operational-amplifier-based circuits with applications to smart skins, IEEE Tran. Ant. Prop. 47, 1330 (1999)

15. S. Tretyakov, Meta-materials with wideband negative permittivity and permeability, Microwave Opt. Technol. Lett. 31, 163 (2001)

16. S. Hrabar, I. Krois, B. Ivsic, D. Zaluski, G. Pavlaković, Active Dispersionless "Plasmonic" Metamaterial - a Step Towards Broadband Cloaking, in: Proc. of the 2008 IEEE AP-S International Symposium and USNC/URSI National Radio Science Meeting (San Diego, 2008), p. 203

17. S. Hrabar, Active non-Foster metamaterials: from intriguing background physics to real-world applications, a plenary talk, in: Proc. on Metamaterials Congress (Sankt Petersburg, 2012)

18. S. Hrabar, Active radiofrequency metamaterial structures Pros, cons and future trends, in: Proc. on Metamaterials Congress (Oxford, 2015)

19. S. Hrabar, Active Dispersionless Metamaterials - a Path towards broadband Cloaking, in: Proc. on 2009 REME Workshop (Madrid, 2009)

20. S. Hrabar, I. Krois, A. Kiricenko, Towards active dispersionless ENZ metamaterial for cloaking applications, J. Metamaterials 4, 89 (2010)

21. B. Okorn, S. Hrabar, I. Krois, Investigation of basic physics of non-Foster negative capacitance in time domain, in: Proc. on ELMAR (Zadar, 2011) p. 373 
22. B. Okorn S. Hrabar, I. Krois, Physically sound model of a non-Foster negative capacitor, Automatika: J. Control Meas. Electron. Comput. Commun. 55, 244 (2017)

23. P.Y. Chen, C. Argyropoulos, A. Alù, Broadening the cloaking bandwidth with non-Foster metasurfaces, Phys. Rev. Lett. 111, 233001 (2013)

24. J.C. Soric, A. Alù, Wideband tunable and non-Foster mantle cloaks, presented at USNC-URSI Nat Radio Sci Meeting, Boulder, CO, USA, 2014

25. S. Hrabar, Z. Sipus, I. Malcic, Broadening of cloaking bandwidth by passive and active techniques, in: D. Werner, D. Kwon (Eds.) A chapter in Transformation Electromagnetics and Metamaterials (Springer, London, 2013)

26. S. Hrabar, I. Krois, I. Bonic, A. Kiricenko, Negative capacitor paves the way to ultra-broadband metamaterials, Appl. Phys. Lett. 99, 25403 (2011)

27. S. Hrabar, I. Krois, I. Bonic, A. Kiricenko, Ultra-broadband simultaneous superluminal phase and group velocities in nonFoster epsilon-near-zero metamaterial, Appl. Phys. Lett. 102, 054081 (2013)

28. J. Long, M. Jacob, D. Sievenpiper, Broadband fast-wave propagation in a non-Foster circuit loaded waveguide, IEEE Trans. Microw. Theory Techn. 62, 789 (2014)

29. A. Niang, A. de Lustrac, S.N. Burokur, Superluminal wave propagation in a non-Foster negative capacitor loaded transmission line, Electron. Lett. 53, 547 (2017)

30. J. Long, Non-Foster Circuit Loaded Periodic Structures for Broadband Fast and Slow Wave Propagation, Ph.D. Thesis, University of California, San Diego, 2015, escholarship.org/ uc/item/1q90m4jj.pdf

31. S. Hrabar, I. Krois, I. Bonic, A. Kiricenko, Non-Foster elements - new path towards broadband ENZ and MNZ metamaterials, in: Proc. on EUCAP, (Prague, 2011), p. 2674

32. G. Eleftheriades, Analysis of bandwidth and loss in negativerefractive-index transmission-line (NRI-TL) media using coupled resonators, IEEE Microw. Wireless Compon. Lett. 17, 412 (2007)

33. S. Hrabar, I. Krois, I., Bonic, A. Kiricenko, D. Muha, Active Reconfigurable Metamaterial Unit Cell Based on Non-Foster Elements, Final Report for Contract FA8655-12-12081, 2013 EOARD / AFRL

34. H.-T. Chen, A.J. Taylor, N. Yu, A review of metasurfaces: physics and applications, Rep. Prog. Phys. 79, 1 (2016)

35. D. Gregoire, C. White, J. Colburn, Wideband artificial magneticconductors loaded with non-Foster negative inductors, IEEE Ant. Wirel. Prop. Lett. 10, 1586 (2011)

36. D.J. Gregoire, C.R. White, J.S. Colburn, A coaxial TEM cell for direct measurement of UHF artificial magnetic conductors, IEEE Ant. Prop. Mag. 54, 251 (2012)

37. J. Long, D. Sievenpiper, Low-profile and low-dispersion artificial impedance surface in the UHF band based on nonFoster circuit loading, IEEE Trans. Ant. Propag. 64, 3003 (2016)

38. J. Mou, Z. Shen, Design and experimental demonstration of non-Foster active absorber, IEEE Trans. Ant. Propag. 65, 696 (2017)

39. J. Mou, Z. Shen, Broadband and thin magnetic absorber with non-Foster metasurface for admittance matching, Sci. Rep. 7, 1 (2017)

40. M. Barbuto, A. Monti, F. Bilotti, A. Toscano, Design of a non-Foster actively loaded SRR and application in metamaterial inspire components, IEEE Trans. Ant. Propag. 61, 1219 (2013)
41. N. Zhu, R.W. Ziolkowski, Broad-bandwidth electrically small antenna augmented with an internal non-Foster element, IEEE Ant. Wirel. Propag. Lett. 11, 1116 (2012)

42. N. Zhu, R.W. Ziolkowski, Broad bandwidth, electrically small, non-Foster element-augmented antenna designs, analyses, and measurements, IEICE Tran. Comm. 96, 2399 (2013)

43. F. Albarracín-Vargas, V. Gonzalez-Posadas, F. Javier Herraíz-Martinez, D. Segovia-Vargas, Design method for actively matched antennas with non-Foster elements, IEE Tran. Ant. Prop. 64, 4118 (2016)

44. H. Mirzaei, G.V. Eleftheriades, A resonant printed monopole antenna with an embedded non-Foster matching network, IEEE Trans. Ant. Prop. 61, 5363 (2013)

45. D.F. Sievenpiper, Superluminal waveguides based on nonFoster circuits for broadband leaky-wave antennas, IEEE Ant. Wirel. Prop. Lett. 10, 231 (2011)

46. D. Muha, S. Hrabar, I. Krois, I. Bonic, A. Kiricenko, D. Zaluski, Design of UHF Microstrip Non-Foster Leaky-wave Antenna, in: Proc. ICECOM (Dubrovnik, 2013), p. 134

47. S. Hrabar, Leaky-wave Antenna based on Non-Foster Metamaterial - is stable operation feasible? in: Proc. on IWAT (Seoul, 2015), p. 7

48. O.O. Tade, Negative impedance converter for antenna matching, PhD thesis, University of Birmingham, 2014, http://etheses.bham.ac.uk/4920/1/Tade14PhD.pdf

49. C.R. White, J.W. May, J.S. Colburn, A variable negativeinductance integrated circuit at UHF frequencies, IEEE Microw. Wireless Compon. Lett. 22, 35 (2012)

50. S. Saadat, H. Aghasi, E. Afshari, H. Mosallaei, Low-power negative inductance integrated circuits for $\mathrm{GHz}$ applications, IEEE Microw. Wireless Compon. Lett. 25, 118 (2015)

51. S.E. Sussman-Fort, Gyrator-based biquad filters and negative impedance converters for microwaves, Int. J. RF Microwave CAE 8, 86 (1998)

52. S. Kolev, B. Delacressonniere, J. Gautier, Using a negative capacitance to increase the tuning range of a varactor diode in mmic technology, IEEE Tran. Microw. Theory Tech. 49, 2425 (2001)

53. D.S. Nagarkoti, Y. Hao, D.P. Steenson, L. Li, E.H. Linfield, K.Z. Rajab, Design of broadband non-Foster circuits based on resonant tunneling diodes, IEEE Ant. Wirel. Propag. Lett. 15, 1398 (2015)

54. H. Mirzaei, G.V. Eleftheriades, Realizing non-Foster reactive elements using negative-group-delay networks, IEEE Trans. Microw. Theory Techn. 61, 4322 (2013)

55. H. Mirzaei, Negative-group-delay and non-Foster electromagnetic structures, Ph.D. dissertation, Dept. Elect. Comput. Eng., Toronto Univ., Toronto, ON, Canada, 2015.

56. H. Mirzaei, G.V. Eleftheriades, Arbitrary-angle squint-free beamforming in series-fed antenna arrays using non-Foster elements synthesized by negative-group-delay networks, IEEE Trans. Ant. Propag. 63, 1997 (2015)

57. T.P. Weldon, J.M.C. Covington, K. Smith R.S. Adams, Performance of digital discrete-time implementations of nonFoster circuit elements, in: Proc. on ISCAS (Lisbon, 2015), p. 2169

58. P.J. Kehoe K.K. Steer T.P. Weldon, Thevenin forms of digital discrete-time non-Foster RC and RL circuits, in: Proc. on IEEE APS/URSI (Fajardo, 2016), pp. 191-192

59. B.I. Popa, S.A. Cummer, An architecture for active metamaterial particles and experimental validation at $\mathrm{RF}$, Microw. Opt. Techn. Lett. 49, 2574 (2007) 
60. Y. Yuan, B.-I. Popa, S.A. Cummer, Zero loss magnetic metamaterials using powered active unit cells, Opt. Express 17, 16135 (2009)

61. E. Ugarte-Muñoz, S. Hrabar, D, Segovia-Vargas, A. Kiricenko, Stability of Non-Foster reactive elements for use in active metamaterials and antennas, IEEE Tran. AP-S 60, $3490(2012)$

62. S. Stearns, Counterintuitive Aspects of Non-Foster Networks, Presentation slides from Adelphi Antenna Workshop on Electrically Small Antennas, Clarksville, MD, July 8-9, 2010

63. S.D. Stearns, Non-Foster Circuits and Stability Theory, in: Proc. on IEEE APS/URSI (Spokane, 2011), p. 1942

64. S.D. Stearns, Incorrect Stability Criteria for Non-Foster Circuits, in: Proc. on IEEE APS/URSI (Chicago, 2012), p. 1

65. S. Tretyakov, S. Maslovski, Veselago materials: what is possible and impossible about the dispersion of the constitutive parameters, IEEE Ant. Prop. Mag. 49, 37 (2007)

66. Q. Tang, H. Xin, Stability analysis of Non-Foster circuit using normalized determinant function, IEEE Tran. Microw. Theory Techn. 65, 3269 (2017)

67. S.E. Sussman-Fort, Matching Network Design Using NonFoster Impedances, Presentation slides, http://www.ieee.li/ pdf/viewgraphs/matching_network_design_non_foster_impedances.pdf

68. S.E. Sussman-Fort, Non-Foster impedance matching of electrically-small antennas, IEEE Trans. Ant. Prop. 57, 2230 (2009)

69. S. Hrabar, Is Stable dispersionless non-Foster DNG Metamaterial Indeed Impossible? in: Proc. on Metamaterial Congress (Copenhagen, 2014), p. 13

70. S. Hrabar, Metamaterial Structures based on "Negative" Elements-What Do We Know After a Decade of Research? in: Proc. on Metamaterial Congress (Rome, 2017) p. 197

71. S.R. Rengarajan, C.R. White, Stability analysis of superluminal waveguides periodically loaded with non-Foster circuits, IEEE Anten. Wireless Prop. Lett. 12, 1303 (2013)

72. J. Loncar, S. Hrabar, D. Muha, Stability of simple lumpeddistributed networks with negative capacitors, IEEE Trans. Ant. Prop. 65, 390 (2017)

73. Y. Fan, Research and Design of Non-Foster Active Metamaterials, Ph.D. Thesis, Queen Mary University, United Kingdom, 2013, http://qmro.qmul.ac.uk/jspui/han dle/123456789/8727
74. K.Z. Rajab, Y. Hao, D. Bao, C.G. Parini, C. Vazquez, J. Philippakis, M. Philippakis, Stability of active magnetoinductive metamaterials, J. Appl. Phys. 108, 054904-1 (2010)

75. K.Z. Rajab, Y.F. Fan, Y. Hao, Characterization of active metamaterials based on negative impedance converters, J. Opt. 14, 114004-1 (2012)

76. S. Saadat, M. Adnan, H. Mosallaei, E. Afshari, Composite metamaterial and metasurface integrated with non-Foster active circuit elements: a bandwidth-enhancement investigation, IEEE Trans. Ant. Propag. 61, 1210 (2013)

77. S.D. Stearns, Stable Band-pass Non- Foster Circuits, in: Proc. on IEEE APS/URSI (Vancouver, 2015), p. 1386

78. S. Hrabar, A. Kiricenko, Towards broadband tunable nonFoster radiating systems, in: Proc. on Metamaterials Congress (Crete, 2016), p. 133

79. S. Hrabar, A. Kiricenko, I. Krois, Antenna-transmitter based on Non-Foster Source, in: Proc. on IEEE APS/URSI (San Diego, 2017), p. 875

80. J. Loncar, S. Hrabar, A. Kiricenko, Stability of MetasurfaceBased Parity-Time Symmetric Systems, in: Proc. on Metamaterial Congress (Crete, 2016), p. 738

81. Y. Ràdi, D.L. Sounas, A. Alù, S.A. Tretyakov, Paritytime symmetric teleportation, Phys. Rev. B 93, 235427 (2016)

82. J Vehmas, S. Hrabar, S. Tretyakov, Omega transmission lines with applications to effective medium models of metamaterials, J. Appl. Phys. 115, 134905-1 (2014)

83. J. Vehmas, S. Hrabar, S. Tretyakov, Transmission lines emulating moving media, N. J. Phys. 16, 1 (2014)

84. D. Zaluski, S. Hrabar, D. Muha, Practical realization of DB metasurface, Appl. Phys. Lett. 104, 234106-1 (2014)

85. D. Zaluški, A. Grbic, S. Hrabar, Analytical and experimental characterization of metasurfaces with normal polarizability, Phys. Rev. 93, 155156-1 (2016)

86. I.V. Lindell, A. Sihvola, Electromagnetic wave reflection from boundaries defined by general linear and local conditions, IEEE Trans. Ant. Prop. 65, 4656 (2017)

87. J. Skaar, Fresnel equations and the refractive index of active media, Phys. Rev. E 73, 026605-1 (2006)

88. A. Fang, Reducing the losses of optical metamaterials, Ph.D. Thesis, Iowa State University, 2013, http://lib.dr.iastate. edu/etd/11807 